Chinese Journal of Organic Chemistry

\title{
6,7-二氧代-4-芳胺香豆素的设计、合成及分子对接研究
}

\author{
王爱玲 ${ }^{a, b}$ 陶 波 ${ }^{*, a}$ 艾纯芝 ${ }^{c}$ 郑学仿 ${ }^{*}, b$ \\ $\left({ }^{a}\right.$ 东北农业大学农学院 哈尔滨 150030$)$ \\ $\left({ }^{b}\right.$ 大连大学辽宁省生物有机化学重点实验室 大连 116622) \\ ( ${ }^{c}$ 中国科学院大连化学物理研究所药品资源研发室 大连 116023)
}

\begin{abstract}
摘要 茶酚-氧位-甲基转移(COMT)酶抑制剂在治疗帕金森病中起到重要作用. 通过对现有 COMT 酶抑制剂托卡朋和 恩托卡朋结构与活性关系分析, 推断含有儿茶酚结构的香豆素类化合物可能具有潜在的 COMT 酶抑制活性, 因此设计 合成了一类新型的 6,7-二氧代-4-芳胺香豆素, 通过理论计算, 研究了此类化合物对 COMT 酶抑制活性. 结果表明, 设 计的 10 种 6,7-二氧代-4-芳胺香豆素与 COMT 酶的对接效果均较好, 其中具有甲氧基乙基保护的儿茶酚结构化合物 6,7二[2-(甲氧基)乙氧]-4-(苯胺)香豆素(6b4)和 6,7-二[2-(甲氧基)乙氧]-4-[(3-乙炔基)苯胺]香豆素(6b5)与 COMT 酶的对接效 果尤为显著.
\end{abstract}

关键词＼cjkstart香豆素; COMT 酶抑制剂; 左旋多巴; SAR; 帕金森病

\section{Design, Synthesis and Molecular Docking Study of 6,7-Dioxo-4-aryl-aminocoumarin}

\author{
Wang, Ailing ${ }^{a, b} \quad$ Tao, Bo ${ }^{*, a} \quad$ Ai, Chunzhi ${ }^{c} \quad$ Zheng, Xuefang ${ }^{*, b}$ \\ $\left({ }^{a}\right.$ College of Agronomy, Northeast Agricultural University, Harbin 150030) \\ ( ${ }^{b}$ Liaoning Key Laboratory of Bio-organic Chemistry, Dalian University, Dalian 116622) \\ ( ${ }^{c}$ Laboratory of Pharmaceutical Resource Discovery, Dalian Institute of Chemical Physics, \\ Chinese Academy of Sciences, Dalian 116023)
}

\begin{abstract}
Catechol- $O$-methyltransferase (COMT) inhibitors play an important role in the treatment of Parkinson's disease (PD). On the basis of the structure-activity relation (SAR) analysis of existing COMT inhibitors, entacapone and tolcapone, it has been derived that coumarin compounds containing catechol structures may have inhibitory bioactivities on COMT. Thus, the novel COMT inhibitors, 6,7-dioxo-4-arylaminocoumarin compounds, were designed, and their inhibitory bioactivities on COMT were investigated by theoretical calculation. The results show that ten 6,7-dioxo-4-aryl-aminocoumarin compounds exhibit good docking effect, especially 6,7-bis(2-methoxyethoxy)-4-phenylamino-2H-chromen-2-one (6b4) and 4-(3-ethynylphenyl)amino-6,7-bis(2-methoxyethoxy)-2H-chromen-2-one (6b5) which have the structure of catechol protected by methoxy ethyl group.

Keywords coumarin; COMT inhibitor; $L$-dopa; SAR; Parkinson's disease
\end{abstract}

帕金森病是继阿尔滋海默病之后第二大神经退行 性疾病 ${ }^{[1]}$, 严重威胁人类的健康. 目前临床上最有效的 治疗药物是左旋多巴 $(L$-dopa). 左旋多巴进入体内后会 被儿茶酚-氧位一甲基转移酶(Catechol-O-Methyltransferase, COMT)和多巴脱羒酶(dopa-decarboxylase, DD)降 解而使其生物利用度降低. COMT 酶广泛分布于哺乳动 物组织中 ${ }^{[2]}$, 在 $\mathrm{Mg}^{2+}$ 的存在下, 通过 $\mathrm{S}_{\mathrm{N}} 2$ 限速机制, 能
够催化辅酶 $S$-腺苷甲硫氨酸(AdoMet)上的甲基转移到 儿茶酚结构的着基上 ${ }^{[3]}$, 从而降低了左旋多巴药效. 为 了提高左旋多巴的生物利用度, 往往左旋多巴给药的同 时需要给病人服用 COMT 酶和 DD 酶抑制剂，以避免左 旋多巴在透过血脑屏障前被这两个酶降解 ${ }^{[4,5]}$. 因此, 研 发具有高临床疗效的 COMT 酶和 DD 酶抑制剂具有重 要意义.

\footnotetext{
*E-mail: botaol@163.com; walwzb@163.com

Received November 3, 2014; revised November 28, 2014; published online December 23, 2014.

Project supported by the National Natural Science Foundation of China (No. 21271036).

国家自然科学基金(No. 21271036)资助项目.
} 
COMT 酶抑制剂的研发目前已经处于第三阶段临 床使用期, Soares-da-Silva 研究小组 ${ }^{[6]}$ 正在 对 opicapone(其结构见表 1)进行各项指标评估. 在过去几 十年里，人们研发了前两代不同结构的 COMT 酶抑制 剂 ${ }^{[7 \sim 10]}$ : 第一代的 Pyrogallol, tropolone 和 gallic acid 等 不仅疗效低而且表现出很强的毒性 ${ }^{[11,12]}$. 在构效关系 (SAR) 的指导下 ${ }^{[13 ~ 15]}, 1989 \sim 1990$ 年, 3 个不同的研究小 组分别研发了恩托卡朋(entacpone)、托卡朋(tolcapone) 和 CGP 28014 等第二代药物. 临床研究表明托卡朋和恩 托卡朋可以安全有效地减少电机波现象, 并可以降低左 旋多巴的剂量. 托卡朋因为肝毒性而被撤市, 从而恩托 卡朋成为临床上唯一可用的 COMT 酶抑制剂. 恩托卡 朋的优势是毒性小，但最大的问题是半衰期太短 $(0.3 \mathrm{~h})$, 疗效也略弱. 因此, 许多研究团队致力于合成疗效更 强、更持久、选择性更强、副作用更低的新 COMT 酶
抑制剂.

经 SAR 分析 ${ }^{[13 \sim 16]}$ 得知, 含有以下结构特征官能团 的 COMT 酶抑制剂具有强的生物活性：首先具有共同 的结构单元儿茶酚(邻二酚), 其次在羟基的邻、对位(结 构见表 1)含有吸电子基团，如硝基或氰基等. 第二代 COMT 酶抑制剂托卡朋和恩托卡朋含有一个共同的药 效基团 5-硝基儿茶酚, 但由于儿茶酚结构中羟基在体内 环境很不稳定，易被甲基化，随即转化为代谢底物，从 而缩短了药物的半衰期、降低了生物利用度，因此有必 要设计一种羟基保护的儿茶酚结构化合物，使羟基不被 甲基化，同时还具备 COMT 酶抑制活性能力; 另外，药 物的毒性直接与抑制剂中硝基结构相关 ${ }^{[17]}$. 综合以上 两点，设计一种含有羟基保护的，不含硝基结构的，同 时差基的邻位或对位含有吸电子基团的儿茶酚类化合 物作为有效的 COMT 酶抑制剂尤为重要. 具有羟基保

表 1 常见的 COMT 酶抑制剂结构

Table 1 The structure of COMT inhibitors

\begin{tabular}{|c|c|c|c|}
\hline 英文名称 & 化学结构式 & 化学命名 & 类别 \\
\hline Pyrogallol & & Benzene-1,2,3-triol & 第一代 \\
\hline Tropolone & & 2-Hydroxycyclohepta-2,4,6-trienone & 第一代 \\
\hline Gallic acid & & 3,4,5-Trihydroxybenzoic acid & 第一代 \\
\hline Tolcapone & & (3,4-Dihydroxy-5-nitrophenyl)( $p$-tolyl)methanone & 第二代 \\
\hline Entacpone & & (E)-2-Cyano-3-(3,4-dihydroxy-5-nitrophenyl)- $N, N$-diethylacrylamide & 第二代 \\
\hline CGP28014 & & (E)- $N^{\prime}$-(6-Hydroxypyridin-2-yl)- $N, N$-dipropylformimidamide & 第二代 \\
\hline $\begin{array}{l}\text { Opicpone/ } \\
\text { BIA9-1067 }\end{array}$ & & $\begin{array}{l}\text { 2,5-Dichloro-3-(5-(3,4-dihydroxy-5-nitrophenyl)-1,2,4- } \\
\text { oxadiazol-3-yl)-4,6-dimethylpyridine 1-oxide }\end{array}$ & 第三代 \\
\hline
\end{tabular}


护的香豆素类化合物结构正好符合以上两点要求. 香豆 素类化合物 ${ }^{[18,19]}$ 在自然界中广泛存在, 所含有的儿茶酚 结构使其可能具有潜在的 COMT 酶抑制活性 ${ }^{[20]}$. 同时, 设想通过理论计算, 此类羟基修饰的香豆素类化合物是 否可以通过占据活性空腔而达到阻碍与底物分子的结 合, 以便延长药物的半衰期、提高生物利用度. 由此, 本 文设计以 3,5-二硝基儿茶酚为对照, 将选定的 6,7-二氧 代-4-芳胺香豆素类化合物对接在 COMT 酶活性空腔中, 从而阻碍与底物分子(多巴胺)的结合, 预测其潜在的生 物活性. 结果表明, 此类化合物均具有较高的生物活性, 其中化合物 $6 \mathrm{~b} 4$ 和 $6 \mathrm{b5}$ 的 COMT 酶抑制活性最佳. 在理 论计算的指导下, 对选定的 6,7-二氧代-4-芳胺香豆素类 化合物进行全合成研究. 以对苯醌为初始原料, 先后经 Thiele 反应、Fries 重排、醚化、合环、氯化/磺酰化、 氨化成功合成了 10 种新型 6,7-二氧代-4-芳胺香豆素.

\section{1 结果与讨论}

\section{1 目标化合物的分子对接研究}

为了验证模型的可靠性, 首先将晶体结构中的所含 的配体抑制剂 3,5-二硝基儿茶酚(3,5-Dinitrocatechol)对 接到 COMT 酶的活性空腔中 (图 1), 结果显示 Hammerhead Score 分值最高的构象可以很好地重现晶 体结构中的构象, 其双羟基朝向 $\mathrm{Mg}^{2+}$ 离子, 且分别与 GLU199、LYS144 以及 ASN170 形成氢键, 而双硝基分 别与两个水分子 $\left(\mathrm{H}_{2} \mathrm{O} 424\right.$ 和 $\left.\mathrm{H}_{2} \mathrm{O} 442\right)$ 形成氢键, 说明我 们的模型是可靠的.
在此基础上，将所合成的香豆素类衍生物 $6 \mathrm{a} 1 \sim$ $6 \mathrm{a} 5$ 和 $6 \mathrm{b1} \sim 6 \mathrm{b5}$ 分别对接到 COMT 酶的活性空腔中, 并同时应用 Hammerhead 打分函数对其相互作用进行评 价, 考察该类化合物与 COMT 酶的亲合作用, 从而预测 其潜在的 COMT 酶抑制活性. 以 $6 \mathbf{b 5}$ 与 COMT 酶的对 接效果(图 2)为例, 结果显示经甲氧基乙基保护的双羟 基背向 $\mathrm{Mg}^{2+}$ 离子, 且和 $\mathrm{H}_{2} \mathrm{O} 424$ 和 $\mathrm{H}_{2} \mathrm{O} 483$ 形成稳定的 氢键，儿茶酚结构的 5-位(香豆素的 2-位)的羰基氧原子 朝向 $\mathrm{Mg}^{2+}$ 离子, 且与酪氨酸 LYS144 中端基的 $\mathrm{NH}_{2}$ 上的 氢原子形成稳定氢键, 对接效果最佳. 对接的打分函数 结果表 2 .

表 2 目标化合物的分子对接打分结果

Table 2 Molecular docking results of target compounds

\begin{tabular}{cc||cc}
\hline 化合物 & $\begin{array}{c}\text { Hammerhead } \\
\text { Score }\end{array}$ & 化合物 & $\begin{array}{c}\text { Hammerhead } \\
\text { Score }\end{array}$ \\
\hline $\mathbf{6 a 1}$ & 4.6093 & $\mathbf{6 b 1}$ & 5.5785 \\
$\mathbf{6 a 2}$ & 5.7015 & $\mathbf{6 b 2}$ & 6.9639 \\
$\mathbf{6 a 3}$ & 5.2663 & $\mathbf{6 b 3}$ & 4.1032 \\
$\mathbf{6 a 4}$ & 4.9840 & $\mathbf{6 b 4}$ & 7.2020 \\
$\mathbf{6 a 5}$ & 5.8650 & $\mathbf{6 b 5}$ & 7.4277 \\
3,5-Dinitrocatechol & 5.6375 & & \\
\hline
\end{tabular}

羟基保护选择两种取代基，第一种为短链甲基保 护, 即 6a 系列, 第二种为长链甲氧基乙基保护, 即 $\mathbf{6 b}$ 系列. 从表 2 的打分结果看, 两个系列的目标化合物与 COMT 酶的亲合作用能力相比, $\mathbf{6 b}$ 系列(除 $\mathbf{6 b 3}$ 外)高于 6a系列. 含有吸电子诱导效应的氯原子的化合物如 6a1, 6b1, 6a3, $6 \mathbf{b 3}$ 与 3,5-二硝基儿茶酚(3,5-Dinitrocatechol)

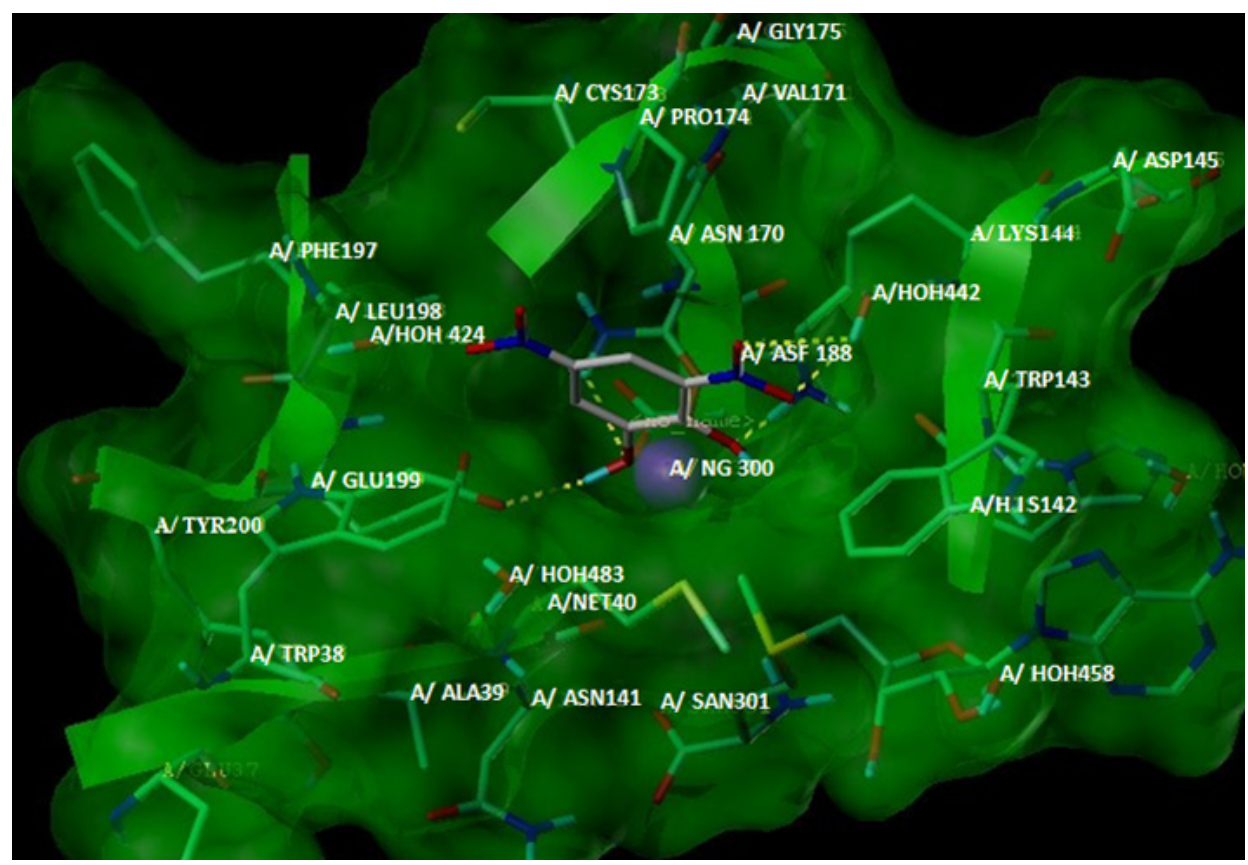

图 1 3,5-Dinitrocatechol 与 COMT 酶间的相互作用

Figure 1 The interaction between 3,5-dinitrocatechol with COMT 


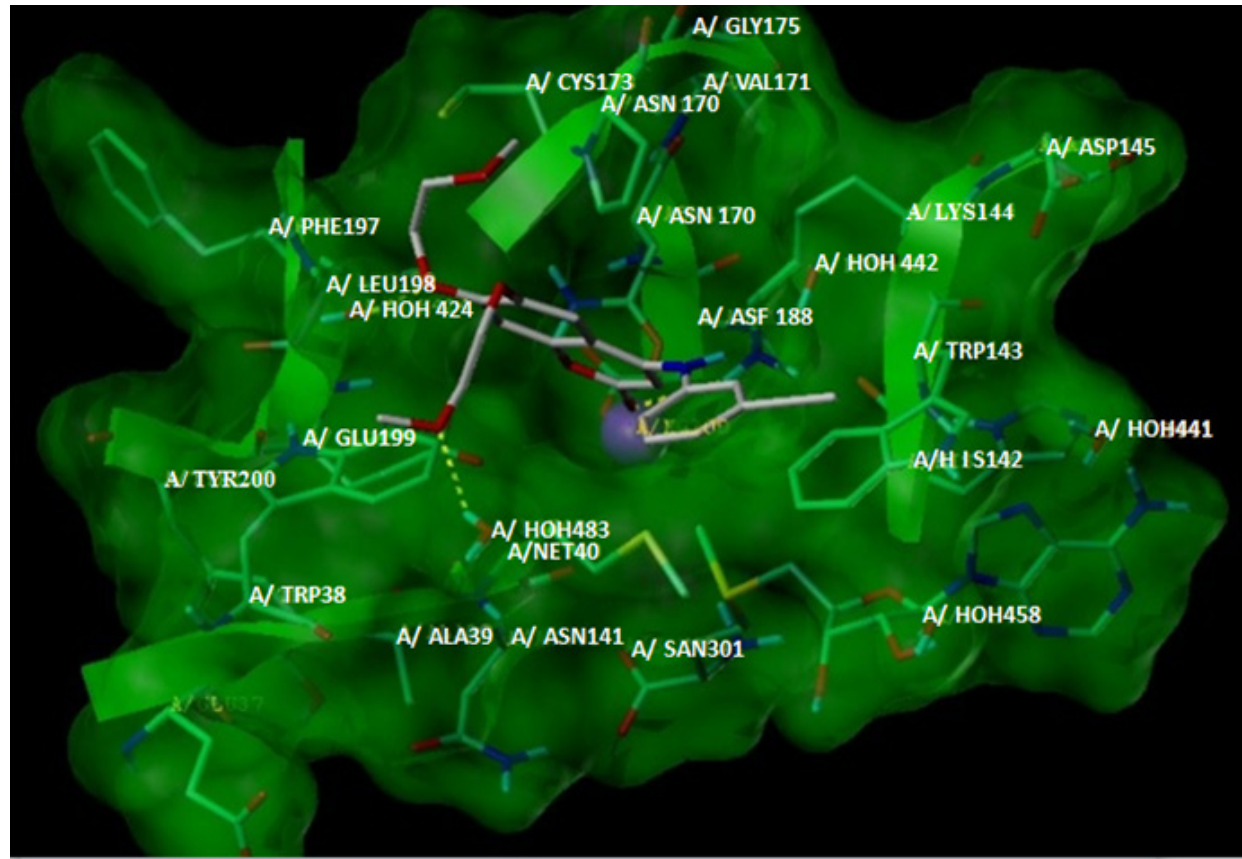

图 2 化合物 $6 \mathbf{b 5}$ (2.2)与 COMT 酶间的相互作用

Figure 2 The interaction between compound $\mathbf{6 b 5}$ with COMT enzymatic

相比, 亲合性略差. 亲合性最好的为含有长链甲氧基乙 基保护的儿茶酚结构化合物香豆素 $6 b 5$ (7.4277), 其次 为 6b4 (7.2020), 分值远高于对照物 3,5-二硝基儿茶酚的 5.6375. 目标化合物的对接打分结果越高说明与 COMT 酶的活性空腔匹配度越好, 相互结合力更强. $6 \mathbf{b}$ 系列的 化合物与空腔体系结合的氢键个数普遍高于对应的 $\mathbf{6 a}$ 系, 与长链的保护基甲氧基乙基上的氧原子个数有关, 增强了与空腔体系 $\mathrm{HOH}$ 形成氢键的能力, 从而结合力 增强, 亲和性提高. 从图 2 可以清晰看出 $6 \mathrm{b5}$ 结构与空 腔体系形成 3 个较强的氢键, 再加上 $6 \mathrm{b5}$ 空间结构上与 COMT 酶空腔的相对吻合, 使得分子对接结果中打分最 高，从而预测其具有潜在的 COMT 酶抑制活性.

\section{2 目标产物的合成}

新型 6,7-二氧代-4-芳胺香豆素 $\mathbf{6 a} \sim \mathbf{6 b}$ 的合成路线 见 Scheme 1. 以对苯醌为起始原料, 在乙酸酐和 $98 \%$ 浓 硫酸作用下经 Thiele 反应得到 1,2,4-三乙酰氧基苯 $(\mathbf{1})^{[11]}$. 化合物 1 在无水三氯化铝 Lewis 酸催化条件下发生 Fries 重排反应, 得到 $2,4,5$-三羟基苯乙酮 $(2)^{[21]}$, 收率为 $60.0 \%$. 此步反应关键在于控制水和浓盐酸的量, 否则 收率极低; 化合物 2 分别与硫酸二甲酯和 1-溴-2-甲氧基 乙烷作用, 在无水碳酸钾碱性条件下, 发生醚化反应, 得到 2-差基-4,5-二甲氧基苯乙酮(3a) ${ }^{[22]}$ 和 1-(2-差基-4,5二(2-甲氧基乙氧)苯基)乙酮(3b); 醚化试剂的量和反应 时间的控制直接影响 $3 \mathrm{a}$ 和 $\mathbf{3 b}$ 的收率, 否则会有一羟基 保护和三羟基保护的副产物生成; 碳酸二乙酯与 $\mathbf{3 a} / \mathbf{3 b}$ 发生关环反应, 以异丙醚为溶剂进行反应, 后经静置、
过滤, 水洗, 盐酸调节 $\mathrm{pH}$ 至 $3.0 \sim 4.0$, 过滤、干燥、乙 醇重结晶, 得到 6,7-二甲氧基-4-羟基香豆素(4a) ${ }^{[23]}$ 和 6,7-二(2-甲氧基乙氧)-4-羟基香豆素(4b). 4a 或 $4 b$ 和三 乙基苄基氯化铵(TEBA)在乙腈体系中经缓慢滴入三氯 氧磷, 发生氯化反应得到 6,7-二甲氧基-4-氯香豆素(5a) 和 6,7-二(2-甲氧基乙氧)-4-氯香豆素(5b). 目标产物 $6 \mathrm{a} 1 \sim 6 \mathrm{a} 4$ 和 $6 \mathrm{~b} 1 \sim 6 \mathrm{~b} 4$ 分别以化合物 $5 \mathrm{a}$ 及 $5 \mathrm{~b}$ 为底物, 与 相应的芳香胺在高温条件下反应得到, 而对目标产物 $6 \mathrm{a} 5$ 和 $6 \mathrm{b5}$ 则不适用. 为解决此问题, 改变合成路径, 通 过对化合物 $4 a$ 和 $4 b$ 的 4 位差基进行活化，进而提高最 后一步的氨化能力. 故选用对甲苯磺酰氯对化合物 4 进 行磺酰化, 后经间乙炔基苯胺氨化, 成功合成期待结构 的目标产物 605 和 $6 \mathrm{b5}$. 但两种方法都存在产率低的缺 点, 其原因可能为反应底物 $5 \mathbf{a}, 5 \mathbf{b}$ 及 $7 \mathbf{a}, 7 \mathbf{b}$ 中的 4 位氯 原子/磺酰基与香豆素环存在不同程度的共轭，所以反 应活性很低. 对于另一反应底物苯胺衍生物, 氮原子中 的孤对电子和苯环共轭, 反应性能也很弱, 所以致使目 标产物的产率较低.

\section{2 结论}

首先，通过理论模型验证，将设计的目标产物对接 到 COMT 酶活性空腔中, 用 Hammerhead 打分函数进行 打分, 以 3,5-二硝基儿茶酚的打分结果作为对照, 结果 表明: 所设计的香豆素类化合物均得到较好的对接结 果, 其中化合物 $6 \mathrm{~b} 4$ 和 $6 \mathrm{b5}$ 的对接得分最高, 高于 3,5二硝基儿茶酚的对接得分，说明 $6 \mathrm{~b} 4$ 和 $6 \mathrm{b5}$ 可能产生良 
<smiles>CC#CC(=O)c1cc(O)c(O)cc1O</smiles>

1 2<smiles></smiles>

4a, 4b $5 a, 5 b$

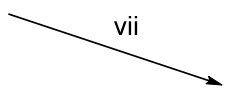

$\mathrm{R}^{1}=\mathrm{Me}(\mathbf{a}), \mathrm{C}_{2} \mathrm{H}_{4} \mathrm{OCH}_{3}$ (b)

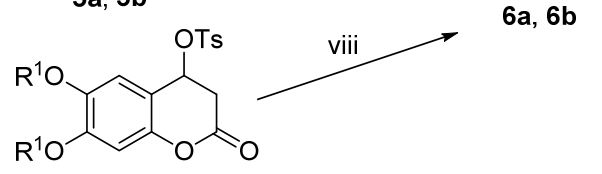

$\mathrm{R}^{2}=2-\mathrm{Cl}(1), 4-\mathrm{CH}_{3}$ (2), 4-Cl (3), $\mathrm{H}(4), 3-\mathrm{C} \equiv \mathrm{CH}$ (5)

Reagents and conditions: (i) $\mathrm{Ac}_{2} \mathrm{O}, \mathrm{H}_{2} \mathrm{SO}_{4}, 40.0 \sim 50.0{ }^{\circ} \mathrm{C}, 86.0 \%$; (ii) $\mathrm{AlCl}_{3}-\mathrm{PhCl}, 80.0 \sim 90.0{ }^{\circ} \mathrm{C}$; $0.5 \mathrm{~h}, 60.0 \%$; (iii) $3 \mathrm{a},\left(\mathrm{CH}_{3}\right)_{2} \mathrm{SO}_{4}, \mathrm{~K}_{2} \mathrm{CO}_{3}$, Acetone, reflux, $1 \mathrm{~h}, 66.4 \%$; (3b) $\mathrm{MeOC}_{2} \mathrm{H}_{5} \mathrm{Br}, \mathrm{K}_{2} \mathrm{CO}_{3}, \mathrm{CH}_{3} \mathrm{CN}$, reflux, $6 \mathrm{~h}, 73.4 \%$; (iv) $\mathrm{CO}\left(\mathrm{OC}_{2} \mathrm{H}_{5}\right)_{2}, \mathrm{Na}$, reflux, Xylene, 72.4\% (4a), 70.8\% (4b); (v) $\mathrm{POCl}_{3}$, TEBA, $90{ }^{\circ} \mathrm{C} 1 \mathrm{~h}, 55.5 \%(5 \mathrm{a}), 54.0 \%(5 \mathrm{~b})$; (vi) Ethylene glycol, $160.0 \sim 180.0{ }^{\circ} \mathrm{C}, 2 \mathrm{~h}, 46.4 \% \sim 56.8 \% ;(v i i) \mathrm{TsCl}, \mathrm{N}\left(\mathrm{C}_{2} \mathrm{H}_{5}\right)_{3}, 20{ }^{\circ} \mathrm{C}$, $97.6 \%$ (7a), $98.1 \%$ (7b); (viii) 3-Ethynylaniline, $\mathrm{K}_{2} \mathrm{CO}_{3}$, Ethanol, reflux, $10.0 \%$ (6a5), 8.6\% (6b5).

图式 1 新型 6,7-二氧代-4-芳胺香豆素 $\mathbf{6 a} \sim \mathbf{6 b}$ 的合成路线

Scheme 1 Synthesis of 6,7-dioxo-4-aryl-aminocoumarins $\mathbf{6 a} \sim \mathbf{6 b}$

好的 COMT 酶抑制活性. 其次, 以对苯醌为原料, 通过 Thiele 反应以 $86 \%$ 的收率得到了 1,2,4-三乙酰氧基苯, 然后在无水 $\mathrm{AlCl}_{3}$ 的催化条件下进行 Fries 重排反应, 得 到了 2,4,5-三羟基苯乙酮, 先后通过硫酸二甲酯和 1-澳2-甲氧基乙烷醚化、碳酸二乙酯环合、三氯氧磷氯化或 经对甲苯磺酰氯先磺酰化后氨化合成了 10 个新型 6,7二氧代-4-芳胺香豆素化合物.

\section{3 实验部分}

\section{1 试剂与仪器}

核磁共振仪(BRUKER ADVANCE $400 \mathrm{MHz}$ ), 高效 液相色谱-质谱联用仪(Shimadzu LC-MS-2010EV, 日本 岛津), 气质联用仪(HP6890/5793, 美国 Hewlett-Packard 公司), X-4 数字显示显微熔点测定仪(巩义市英峪予华 仪器厂), 傅立叶变换红外光谱仪 (美国尼高力, 550), RE-52C 旋转蒸发仪(巩义市英峪予华仪器厂), DF-101S 集热式恒温加热磁力搅拌器(巩义市英峪予华仪器厂). 试剂为分析纯或化学纯, 使用前未经进一步纯化.

\section{2 目标化合物的分子模建预测 COMT 酶抑制活性的 评估}

采用 Sybyl 模块中的 Sur-flexdock 方法, 选择 COMT 酶的晶体结构(PDB code: 3BWM), 应用原型位 点的方法生成其活性空腔, 以将所合成的化合物对接到 活性空腔当中. 对接过程中每个化合物生成 20 种构象, 应用 Hammerhead 打分函数对其进行打分, 选择排序第 一的为其优势构象. 小分子化合物的构象搜索和能量优
化是在 Sybyl 程序中的 Tripos 力场(Sybyl7.3 Tripos Inc.) 进行的, 利用 Powell 联合梯度算法在 $0.001 \mathrm{kcal} / \mathrm{mol}$ 的

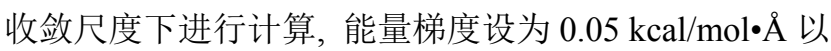
获取最稳定构象，原子电荷来用 Gasteiger-Huckel 方法 计算获得.

\section{3 实验过程}

\subsubsection{1,2,4-三乙酰氧基苯(1)的合成}

取 $150.0 \mathrm{~mL}$ 乙酸酎和 $4.0 \mathrm{~mL} 98 \%$ 浓硫酸于 40.0 $50.0{ }^{\circ} \mathrm{C}$ 下, 分批加入 $50.0 \mathrm{~g}(0.46 \mathrm{~mol})$ 对苯醌, 待全部 加完后, 保温 $1.5 \mathrm{~h}$. 冷却至 $25.0{ }^{\circ} \mathrm{C}$, 倒入 $600.0 \mathrm{~mL}$ 水 中, 剧烈搅拌, 过滤析出的固体, 乙醇重结晶得白色固 体 $\mathbf{1}^{[11]} 100.0 \mathrm{~g}$, 收率 86.0\%. m.p. $97.0 \sim 98.0{ }^{\circ} \mathrm{C}$ (文献值 [11]: 96.5 97.0 $\left.{ }^{\circ} \mathrm{C}\right) ;{ }^{1} \mathrm{H}$ NMR $\left(\mathrm{CDCl}_{3}, 400 \mathrm{MHz}\right) \delta: 2.28$ (s, $9 \mathrm{H}), 7.00 \sim 7.04(\mathrm{~m}, 2 \mathrm{H}), 7.18(\mathrm{~d}, J=8 \mathrm{~Hz}, 1 \mathrm{H})$.

\subsubsection{2,4,5-三羟基苯乙酮(2)的合成}

取 $53.0 \mathrm{~g}(0.4 \mathrm{~mol})$ 无水三氯化铝和 $70.0 \mathrm{~mL}$ 氯苯, 保持 $30.0 \sim 40.0{ }^{\circ} \mathrm{C}$ 下, 缓慢加入 $25.0 \mathrm{~g}(0.10 \mathrm{~mol}) 1,2,4-$ 三乙酰氧基苯的 $80.0 \mathrm{~mL}$ 氯苯悬浮液, 加完后温度升至 $80.0 \sim 90.0{ }^{\circ} \mathrm{C}$, 保温 $0.5 \mathrm{~h}$, 冷却至室温. 反应液倒入 $200.0 \mathrm{~g}$ 冰和 $50.0 \mathrm{~mL}$ 浓盐酸中, 搅拌 $1.0 \mathrm{~h}$ 后过滤, 滤饼 用无水乙醇重结晶, 得 $10.0 \mathrm{~g}$ 淡黄色针状固体 $\mathbf{2}^{[21]}$, 收 率 $60.0 \%$. m.p. $209.0 \sim 210.0{ }^{\circ} \mathrm{C}$ (文献值 ${ }^{[21]}: 204.0 \sim$ $206.0{ }^{\circ} \mathrm{C}$ ). ${ }^{1} \mathrm{H}$ NMR (DMSO- $\left.d_{6}, 400 \mathrm{MHz}\right) \delta: 2.45$ (s, 3H), $6.28(\mathrm{~s}, 1 \mathrm{H}), 7.15(\mathrm{~s}, 1 \mathrm{H}), 12.23(\mathrm{~s}, 1 \mathrm{H})$.

\subsubsection{2-羟基-4,5-二甲氧基苯乙酮 $(3 \mathbf{a})$ 的合成}

取 $8.0 \mathrm{~g}$ (47.60 mmol) 2,4,5-三羟基苯乙酮、 $19.7 \mathrm{~g}$ 
$(142.73 \mathrm{mmol})$ 无水碳酸钾和 $70.0 \mathrm{~mL}$ 丙酮, 升温至 $60.0{ }^{\circ} \mathrm{C}$, 滴加 $13.2 \mathrm{~g}(104.67 \mathrm{mmol})$ 硫酸二甲酯, 加完后 反应 $1.0 \mathrm{~h}$, 冷却至室温, 过滤, 滤液减压浓缩得到棕褐 色的固体, 乙醇重结晶, 得棕褐色固体 $3 \mathrm{a}^{[22]} 6.2 \mathrm{~g}$, 收率 $66.4 \%$. m.p. $114.0 \sim 116.0{ }^{\circ} \mathrm{C}$ (文献值 ${ }^{[22]}: 114.0 \sim$ $\left.116.0{ }^{\circ} \mathrm{C}\right) ;{ }^{1} \mathrm{H} \mathrm{NMR}\left(\mathrm{CDCl}_{3}, 400 \mathrm{MHz}\right) \delta: 2.57$ (s, 3H), $3.87(\mathrm{~s}, 3 \mathrm{H}), 3.92(\mathrm{~s}, 3 \mathrm{H}), 6.46(\mathrm{~s}, 1 \mathrm{H}), 7.06(\mathrm{~s}, 1 \mathrm{H})$

3.3.4 1-[2-羟基-4,5-二(2-甲氧基乙氧)苯基]乙酮 $(3 \mathbf{b})$ 的合成

取 $8.4 \mathrm{~g}(0.05 \mathrm{~mol}) 2,4,5$-三羟基苯乙酮、 $20.7 \mathrm{~g}(0.15$ mol)无水碳酸钾和 $80.0 \mathrm{~mL}$ 乙腈, 升温至 $85.0{ }^{\circ} \mathrm{C}$, 缓慢 滴加 $12.0 \mathrm{~mL}$ 1-澳-2-甲氧基乙烷和 $10.0 \mathrm{~mL}$ 丙酮的混合 液, 滴加完后反应 $6.0 \mathrm{~h}$. 冷却, 溶液过滤, 乙酸乙酯洗 涤滤饼, 将滤液旋干, 柱分离 $[V$ (石油醚) : $V$ (乙酸乙 酯 $)=5: 1$ ], 得 $10.4 \mathrm{~g}$ 白色固体, 收率 $73.4 \%$. m.p. $51.0 \sim 52.0{ }^{\circ} \mathrm{C} ;{ }^{1} \mathrm{H}$ NMR $\left(\mathrm{CDCl}_{3}, 400 \mathrm{MHz}\right) \delta: 2.51(\mathrm{~s}$, $3 \mathrm{H}), 3.43$ (s, 6H), 3.71 (t, $J=4 \mathrm{~Hz}, 2 \mathrm{H}), 3.77$ (t, $J=4 \mathrm{~Hz}$, $2 \mathrm{H}), 4.09$ (t, $J=4 \mathrm{~Hz}, 2 \mathrm{H}), 4.15$ (t, $J=4 \mathrm{~Hz}, 2 \mathrm{H}), 6.41$ (s, 1H), $7.25(\mathrm{~s}, 1 \mathrm{H}), 12.56(\mathrm{~s}, 1 \mathrm{H}) ;{ }^{13} \mathrm{C}$ NMR $\left(\mathrm{CDCl}_{3}, 100\right.$ MHz) $\delta: 26.50,59.31,59.38,68.37,70.64,70.93,71.51$, $101.69,112.36,118.18,141.07,157.59,160.72,202.40$; IR (KBr) v: 2925, 1621, 1511, 1376, 1258, 1119, $812 \mathrm{~cm}^{-1}$; GC-MS (EI, $70 \mathrm{eV}$ ) $m / z: 284[\mathrm{M}]^{+}, 226,207,194,179$, 167, 153, 137, 124, 109, 95, 73, 59, 43, 29.

3.3.5 6,7-二甲氧基-4-羟基香豆素(4a)和 6,7-二(2-甲 氧基乙氧)-4-羟基香豆素( $4 \mathbf{b})$ 的合成

$50.0 \mathrm{~mL}$ 二甲苯中加入 $2.1 \mathrm{~g}(0.09 \mathrm{~mol})$ 金属钠, 升 温至回流温度, 然后缓慢加入 $0.03 \mathrm{~mol} \mathbf{3 a}$ 或 $\mathbf{3 b}$, 滴加 $0.15 \mathrm{~mol}$ 的碳酸二乙酯, 滴加完毕后, 反应 $3.0 \mathrm{~h}$, 冷却 至室温, 加入 $200.0 \mathrm{~mL}$ 异丙醚摚拌 $0.5 \mathrm{~h}$ 后静置、过滤, 滤饼溶入 $50.0 \mathrm{~mL}$ 水中, 盐酸调节 $\mathrm{pH}$ 至 $3.0 \sim 4.0$, 过滤, 干燥, 乙醇重结晶.

4a: 收率 72.4\%. m.p. $266.0 \sim 267.0{ }^{\circ} \mathrm{C}$ (文献值 ${ }^{[23]}$ : $278.0{ }^{\circ} \mathrm{C}$ ); ${ }^{1} \mathrm{H}$ NMR (DMSO- $\left.d_{6}, 400 \mathrm{MHz}\right) \delta: 3.81(\mathrm{~s}, 3 \mathrm{H}$ ), $3.85(\mathrm{~s}, 3 \mathrm{H}), 5.47(\mathrm{~s}, 1 \mathrm{H}), 7.02(\mathrm{~s}, 1 \mathrm{H}), 7.18(\mathrm{~s}, 1 \mathrm{H}), 12.40$ (s, 1H).

4b: 收率 70.8\%. m.p. $236.0 \sim 237.0{ }^{\circ} \mathrm{C} ;{ }^{1} \mathrm{H}$ NMR (DMSO- $\left.d_{6}, 400 \mathrm{MHz}\right) \delta: 3.38(\mathrm{~s}, 6 \mathrm{H}), 3.67 \sim 3.70(\mathrm{~m}$, 4H), 4.12 (t, $J=4 \mathrm{~Hz}, 2 \mathrm{H}), 4.20$ (t, $J=4 \mathrm{~Hz}, 2 \mathrm{H}), 5.46$ (s, $1 \mathrm{H}), 7.03(\mathrm{~s}, 1 \mathrm{H}), 7.21(\mathrm{~s}, 1 \mathrm{H}), 12.34(\mathrm{~s}, 1 \mathrm{H}) ;{ }^{13} \mathrm{C} \mathrm{NMR}$ (DMSO- $\left.d_{6}, 100 \mathrm{MHz}\right) \delta: 58.87,58.90,68.96,69.12,70.70$, $70.92,89.41,101.73,106.39,108.40,145.45,150.08$, 153.22, 163.11, 166.55; IR (KBr) v: 2921, 1711, 1617, $1486,1376,1315,1204,812 \mathrm{~cm}^{-1}$; MS (API-ES) $m / z$ : $311.0[\mathrm{M}+1]^{+}, 312.0[\mathrm{M}+2]^{+}$.
3.3.6 6,7-二甲氧基-4-氯香豆素(5a)和 6,7-二(2-甲氧 基乙氧)-4-氯香豆素 $(5 \mathbf{b})$ 的合成

取 $4.50 \mathrm{mmol} \mathbf{4 a}$ 或 $\mathbf{4 b}$ 、三乙基苄基氯化铵(TEBA) $4.1 \mathrm{~g}(18.00 \mathrm{mmol})$ 和乙腈 $20.0 \mathrm{~mL}$, 升温至 $40.0{ }^{\circ} \mathrm{C}$ 搅拌 $10.0 \mathrm{~min}$, 缓慢滴加入 $1.9 \mathrm{~mL}(20.00 \mathrm{mmol})$ 的三氯氧磷, 滴加完成后保温反应 $0.5 \mathrm{~h}$, 然后升温至 $90.0{ }^{\circ} \mathrm{C}$, 减压 蒸馏除去溶剂, 加入 $20.0 \mathrm{~mL}$ 水, 溶液室温摚拌 $3.0 \mathrm{~h}$, 收集析出的固体, 柱分离纯化 ${ }^{[24]}$.

5a: 收率 55.5\%. m.p. 173.0 174.0 ${ }^{\circ} \mathrm{C} ;{ }^{1} \mathrm{H}$ NMR (DMSO- $\left.d_{6}, 400 \mathrm{MHz}\right) \delta: 3.87$ (s, 3H), 3.90 (s, 3H), 6.72 (s, $1 \mathrm{H}), 7.18 \sim 7.20(\mathrm{~m}, 2 \mathrm{H}) ;{ }^{13} \mathrm{C}$ NMR (DMSO- $d_{6}, 100 \mathrm{MHz}$ ) $\delta: 56.04,56.49,100.42,105.26,109.71,111.89,146.39$, 148.49, 148.51, 153.88, 158.86; IR (KBr) v: 1707, 1618, 1507, 1384, 1278, $837 \mathrm{~cm}^{-1}$; GC-MS (EI, $70 \mathrm{eV}$ ) m/z: $240.0[\mathrm{M}]^{+}$.

5b: 收率 54.0\%. m.p. $71.0 \sim 73.0{ }^{\circ} \mathrm{C} ;{ }^{1} \mathrm{H}$ NMR $\left(\mathrm{CDCl}_{3}, 400 \mathrm{MHz}\right) \delta: 3.48(\mathrm{~s}, 6 \mathrm{H}), 3.78 \sim 3.83(\mathrm{~m}, 4 \mathrm{H})$, $4.20 \sim 4.23(\mathrm{~m}, 4 \mathrm{H}), 6.46(\mathrm{~s}, 1 \mathrm{H}), 6.89(\mathrm{~s}, 1 \mathrm{H}), 7.31(\mathrm{~s}$, $1 \mathrm{H}) ;{ }^{13} \mathrm{C} \mathrm{NMR}\left(\mathrm{CDCl}_{3}, 100 \mathrm{MHz}\right) \delta: 59.43,59.45,69.13$, $69.75,70.70,71.09,101.53,109.23,111.02,112.41$, 143.58, 146.39, 149.36, 154.26, 159.87; IR (KBr) v: 1711, 1617, 1486, 1376, 1315, 1204, $812 \mathrm{~cm}^{-1}$; GC-MS (EI, 70 eV) $m / z: 328[\mathrm{M}]^{+}$.

\subsection{7 目标化合物 $6 \mathrm{a} 1 \sim 6 \mathrm{a} 4$ 和 $6 \mathrm{~b} 1 \sim 6 \mathrm{~b} 4$ 的合成}

$5.00 \mathrm{mmol} \mathrm{5}$ 、三乙胺 $(6.00 \mathrm{mmol}) 、$ 相应的芳香胺 (6.00 mmol)和 $5.0 \mathrm{~mL}$ 乙二醇, 升温至 $160.0 \sim 180.0{ }^{\circ} \mathrm{C}$ 反应 $2.0 \mathrm{~h}$, 自然冷却至室温后, 加入 $25.0 \mathrm{~mL}$ 水, 搅拌 $10 \mathrm{~min}$, 静置片刻, 过滤, 无水乙醇重结晶 ${ }^{[25]}$, 收率 $46.4 \sim 56.8 \%$.

4-(2-氯苯胺基)-6,7-二甲氧基香豆素(6a1): 收率 54.3\%. m.p. $304.0 \sim 305.0{ }^{\circ} \mathrm{C} ;{ }^{1} \mathrm{H}$ NMR (DMSO- $d_{6}, 400$ MHz) $\delta: 3.88(\mathrm{~s}, 6 \mathrm{H}), 5.29(\mathrm{~s}, 1 \mathrm{H}), 7.02(\mathrm{~s}, 1 \mathrm{H}), 7.34 \sim$ $7.39(\mathrm{~m}, 2 \mathrm{H}), 7.45(\mathrm{~s}, 1 \mathrm{H}), 7.50 \sim 7.54(\mathrm{~m}, 1 \mathrm{H}), 7.67(\mathrm{~s}$, 1H), 9.20 (brs, $1 \mathrm{H}$ ); ${ }^{13} \mathrm{C}$ NMR (DMSO- $\left.d_{6}, 100 \mathrm{MHz}\right) \delta$ : $56.07,56.25,83.47,100.42,104.02,105.99,123.23$, $124.35,125.44,131.09,133.60,140.01,145.48,149.16$, 152.22, 152.61, 161.87; IR (KBr) v: 3290, 1661, 1585,1531, $853 \mathrm{~cm}^{-1}$; MS (API-ES) m/z: $332.2[\mathrm{M}+1]^{+}$; HRMS (ESI) calcd for $\mathrm{C}_{17} \mathrm{H}_{15} \mathrm{ClNO}_{4}[\mathrm{M}+\mathrm{H}]^{+}: 332.0611$, found 332.0693 .

4-(4-甲基苯胺基)-6,7-二甲氧基香豆素(6a2): 收率 $56.8 \%$. m.p. $289.0 \sim 291.0{ }^{\circ} \mathrm{C} ;{ }^{1} \mathrm{H}$ NMR (DMSO- $d_{6}, 400$ MHz) $\delta: 2.35$ (s, 3H), $3.86(\mathrm{~s}, 3 \mathrm{H}), 5.07(\mathrm{~s}, 1 \mathrm{H}), 6.98(\mathrm{~s}$, 1H), 7.23 (d, $J=8 \mathrm{~Hz}, 2 \mathrm{H}), 7.29$ (d, $J=8 \mathrm{~Hz}, 2 \mathrm{H}), 9.13$ (brs, $1 \mathrm{H}$ ); ${ }^{13} \mathrm{C}$ NMR (DMSO- $\left.d_{6}, 100 \mathrm{MHz}\right) \delta: 21.54,56.90$, 
$57.13,82.86,101.13,104.81,106.80,125.98,130.66$, 136.03, 136.16, 146.09, 153.09, 153.71, 162.61; IR (KBr) $v: 3276,1650,1535,1511,1384,1237,800 \mathrm{~cm}^{-1}$; MS (API-ES) $m / z: 311.9\left[^{[\mathrm{M}}\right]^{+}$; HRMS (ESI) calcd for $\mathrm{C}_{18} \mathrm{H}_{18} \mathrm{NO}_{4}[\mathrm{M}+\mathrm{H}]^{+}: 312.1158$, found 312.1239.

4-(4-氯苯胺基)-6,7-二甲氧基香豆素 (6a3): 收率 49.6\%. m.p. $307.0 \sim 309.0{ }^{\circ} \mathrm{C} ;{ }^{1} \mathrm{H}$ NMR (DMSO- $d_{6}, 400$ MHz) $\delta: 3.87(\mathrm{~s}, 6 \mathrm{H}), 5.21(\mathrm{~s}, 1 \mathrm{H}), 7.01(\mathrm{~s}, 1 \mathrm{H}), 7.40(\mathrm{~d}$, $J=8.4 \mathrm{~Hz}, 2 \mathrm{H}), 7.54$ (d, $J=8.4 \mathrm{~Hz}, 2 \mathrm{H}), 7.68$ (s, 1H), 9.20 (brs, $1 \mathrm{H}) ;{ }^{13} \mathrm{C}$ NMR (DMSO- $\left.d_{6}, 100 \mathrm{MHz}\right) \delta: 56.72,56.94$, $83.65,101.08,104.78,106.68,127.31,130.12,130.35$, 137.98, 146.15, 149.81, 153.17, 153.27, 162.56; IR (KBr) $v: 3256,1650,1531,1507,1380 \mathrm{~cm}^{-1}$; MS (API-ES) $m / z$ : $331.9[\mathrm{M}]^{+}$.

4-苯胺基-6,7-二甲氧基香豆素(6a4): 收率 46.4\%. m.p. $184.0 \sim 186.0{ }^{\circ} \mathrm{C} ;{ }^{1} \mathrm{H}$ NMR (DMSO- $\left.d_{6}, 400 \mathrm{MHz}\right) \delta$ : $3.87(\mathrm{~s}, 6 \mathrm{H}), 5.17(\mathrm{~s}, 1 \mathrm{H}), 7.00(\mathrm{~s}, 1 \mathrm{H}), 7.31 \sim 7.38(\mathrm{~m}$, $3 \mathrm{H}), 7.50(\mathrm{~m}, 2 \mathrm{H}), 7.71(\mathrm{~s}, 1 \mathrm{H}), 9.20$ (brs, $1 \mathrm{H}) ;{ }^{13} \mathrm{C} \mathrm{NMR}$ (DMSO- $\left.d_{6}, 100 \mathrm{MHz}\right) \delta: 56.70,56.94,89.09,101.05$, $104.80,106.75,125.83,126.59,130.16,138.89,146.13$, 149.80, 153.19, 153.49, 162.65; IR (KBr) v: 3252, 1646, 1544, 1503, 1388, $1237 \mathrm{~cm}^{-1}$; MS (API-ES) $\mathrm{m} / \mathrm{z}: 298.1$ $[\mathrm{M}+1]^{+}$; HRMS (ESI) calcd for $\mathrm{C}_{17} \mathrm{H}_{16} \mathrm{NO}_{4}[\mathrm{M}+\mathrm{H}]^{+}$: 298.1001, found 298.1082.

4-(2-氯苯胺基)-6,7-二(2-甲氧基乙氧)香豆素 (6b1): 收率 52.7\%. m.p. 202.0 204.0 ${ }^{\circ} \mathrm{C} ;{ }^{1} \mathrm{H}$ NMR (DMSO- $d_{6}$, $400 \mathrm{MHz}) \delta: 3.33(\mathrm{~s}, 6 \mathrm{H}), 3.69 \sim 3.72(\mathrm{~m}, 4 \mathrm{H}), 4.15 \sim$ $4.22(\mathrm{~m}, 4 \mathrm{H}), 5.27(\mathrm{~s}, 1 \mathrm{H}), 7.03(\mathrm{~s}, 1 \mathrm{H}), 7.30 \sim 7.51(\mathrm{~m}$, $4 \mathrm{H}), 7.68(\mathrm{~s}, 1 \mathrm{H}), 9.19$ (brs, $1 \mathrm{H}) ;{ }^{13} \mathrm{C}$ NMR (DMSO- $d_{6}$, $100 \mathrm{MHz}) \delta: 58.79,68.79,69.18,70.57,70.73,84.06$, $102.05,106.80,123.67,124.80,125.90,131.57,134.10$, $140.55,145.16,149.76,152.63,152.71,162.35$; IR (KBr) $v: 3244,1642,1531,1507,1380,1262 \mathrm{~cm}^{-1}$; MS (API-ES) $m / z$ : $441.9[\mathrm{M}+\mathrm{Na}]^{+}$; HRMS (ESI) calcd for $\mathrm{C}_{21} \mathrm{H}_{23} \mathrm{Cl}-$ $\mathrm{NO}_{6}[\mathrm{M}+\mathrm{H}]^{+}:$420.1136, found 420.1222 .

4-(4-甲基苯胺基)-6,7-二(2-甲氧基乙氧)香豆素 (6b2): 收率 51.8\%. m.p. 193.0 194.0 ${ }^{\circ} \mathrm{C} ;{ }^{1} \mathrm{H}$ NMR (DMSO- $\left.d_{6}, 400 \mathrm{MHz}\right) \delta: 2.34(\mathrm{~s}, 3 \mathrm{H}), 3.33(\mathrm{~s}, 6 \mathrm{H}), 3.69 \sim$ $3.72(\mathrm{~m}, 4 \mathrm{H}), 4.21(\mathrm{~m}, 4 \mathrm{H}), 5.09(\mathrm{~s}, 1 \mathrm{H}), 7.00(\mathrm{~s}, 1 \mathrm{H}), 7.23$ (d, $J=8 \mathrm{~Hz}, 2 \mathrm{H}), 7.29$ (d, $J=8 \mathrm{~Hz}, 2 \mathrm{H}), 7.74(\mathrm{~s}, 1 \mathrm{H}), 9.11$ (brs, $1 \mathrm{H}$ ); ${ }^{13} \mathrm{C}$ NMR (DMSO- $\left.d_{6}, 100 \mathrm{MHz}\right) \delta: 21.24,58.93$, $63.44,68.88,69.25,70.88,82.76,102.14,106.79,106.96$, $125.85,130.60,135.95,136.16,145.26,149.83,152.65$, 153.59, 162.63; IR (KBr) v: 3293, 1650, 1535, 1511, 1380, 1262, $1127 \mathrm{~cm}^{-1}$; MS (API-ES) $m / z: 400.0[\mathrm{M}+1]^{+}$;
HRMS (ESI) calcd for $\mathrm{C}_{22} \mathrm{H}_{26} \mathrm{NO}_{6}[\mathrm{M}+\mathrm{H}]^{+}$: 400.1682, found 400.1766 .

4-(4-氯苯胺基)-6,7-二(2-甲氧基乙氧)香豆素 $(6 \mathbf{b} 3$ ): 收率 48.1\%. m.p. 237.0 239.0 ${ }^{\circ} \mathrm{C} ;{ }^{1} \mathrm{H}$ NMR (DMSO- $d_{6}$, $400 \mathrm{MHz}) \delta: 3.35(\mathrm{~s}, 6 \mathrm{H}), 3.50 \sim 3.90(\mathrm{~m}, 4 \mathrm{H}), 4.19 \sim 4.44$ (m, 4H), $5.22(\mathrm{~s}, 1 \mathrm{H}), 7.03(\mathrm{~s}, 1 \mathrm{H}), 7.28 \sim 7.52(\mathrm{~m}, 5 \mathrm{H})$, $7.72(\mathrm{~s}, 1 \mathrm{H}), 9.16$ (brs, $1 \mathrm{H}$ ); ${ }^{13} \mathrm{C}$ NMR (DMSO- $d_{6}, 100$ MHz) $\delta$ : 58.94, 68.90, 69.30, 70.72, 70.88, 83.69, 102.14, 106.88, 127.22, 130.09, 130.30, 137.97, 145.28, 149.88, $152.79,152.99,162.51$; IR (KBr) v: 3244, 1650, 1535, 1499, 1380, $1262 \mathrm{~cm}^{-1}$; MS (API-ES) $m / z: 419.9[\mathrm{M}+1]^{+}$; HRMS (ESI) calcd for $\mathrm{C}_{21} \mathrm{H}_{23} \mathrm{ClNO}_{6}[\mathrm{M}+\mathrm{H}]^{+}: 420.1136$, found 420.1220 .

4-苯胺基-6,7-二(2-甲氧基乙氧)香豆素(6b4)：收率 50.4\%. m.p. $213.0 \sim 215.0{ }^{\circ} \mathrm{C} ;{ }^{1} \mathrm{H}$ NMR (DMSO- $d_{6}, 400$ MHz) $\delta: 3.34(\mathrm{~s}, 6 \mathrm{H}), 3.71 \sim 3.73(\mathrm{~m}, 4 \mathrm{H}), 4.18 \sim 4.23(\mathrm{~m}$, 4H), $5.18(\mathrm{~s}, 1 \mathrm{H}), 7.03(\mathrm{~s}, 1 \mathrm{H}), 7.28 \sim 7.52(\mathrm{~m}, 5 \mathrm{H}) 7.75$ (s, $1 \mathrm{H}), 9.18(\mathrm{~s}, 1 \mathrm{H}) ;{ }^{13} \mathrm{C}$ NMR (DMSO- $\left.d_{6}, 100 \mathrm{MHz}\right) \delta$ : 58.94, 68.89, 69.26, 70.73, 70.88, 83.12, 102.14, 106.80, $106.95,125.75,126.54,130.14,138.87,145.27,149.86$, 152.71, 153.34, 162.62; IR (KBr) v: 3235, 1650, 1535, $1503,1388,1270 \mathrm{~cm}^{-1}$; MS (API-ES) $m / z: 386.0[\mathrm{M}+1]^{+}$.

\subsection{8 化合物 $7 \mathbf{a}$ 和 $7 \mathbf{b}$ 的合成}

取化合物 $5.0 \mathrm{mmol}(4), 6 \mathrm{mmol}$ 三乙胺加入 $10.0 \mathrm{~mL}$ 二氯甲烷中, 将 $6.0 \mathrm{mmol}$ 的对甲苯磺酰氯( $\mathrm{TsCl})$ 用 10.0 $\mathrm{mL}$ 的二氯甲烷溶解后室温下滴加, TLC $\left(\mathrm{CHCl}_{3}\right.$ : $\left.\mathrm{CH}_{3} \mathrm{OH}=1: 11, \mathrm{~V} / \mathrm{V}\right)$ 监测原料无剩余后停止反应, 用等 体积的碳酸氢钾水溶液洗涤, 有机层用无水硫酸镁干 燥, 过滤, 旋干滤液得 $7 \mathbf{a}$ 和 $\mathbf{7} \mathbf{b}^{[26]}$, 未进行进一步纯化, 直接用于下步反应.

6,7-二甲氧基-4-对甲苯磺酰基香豆素(7a): 收率 97.6\%. m.p. $157.0 \sim 158.0{ }^{\circ} \mathrm{C} ;{ }^{1} \mathrm{H}$ NMR $\left(\mathrm{CDCl}_{3}, 400\right.$ MHz) $\delta: 2.46$ (s, 3H), 3.86 (s, 3H), $3.96(\mathrm{~s}, 3 \mathrm{H}), 6.10(\mathrm{~s}$, $1 \mathrm{H}), 6.79(\mathrm{~s}, 1 \mathrm{H}), 6.91(\mathrm{~s}, 1 \mathrm{H}), 7.38$ (d, $J=8 \mathrm{~Hz}, 2 \mathrm{H}), 7.89$ $(\mathrm{d}, J=8 \mathrm{~Hz}, 2 \mathrm{H}) ;{ }^{13} \mathrm{C} \mathrm{NMR}\left(\mathrm{CDCl}_{3}, 100 \mathrm{MHz}\right) \delta: 22.01$, $56.54,56.69,100.10,101.51,103.19,107.41,128.62$, $130.59,131.97,146.74,147.04,149.96,154.21,158.35$, 161.68; IR (KBr) v: 2925, 1736, 1621, 1564, 1507, 1376, 1147, 1000, 927, $816 \mathrm{~cm}^{-1}$; MS (ESI) $m / z: 377.0[\mathrm{M}+$ $\mathrm{H}]^{+}$;

6,7-二(2-甲氧基乙氧)-4-对甲苯磺酰基香豆(7b)：收 率 98.1\%. m.p. 96.0 97.0 ${ }^{\circ} \mathrm{C} ;{ }^{1} \mathrm{H}$ NMR $\left(\mathrm{CDCl}_{3}, 400\right.$ MHz) $\delta: 2.46$ (s, 3H), 3.45 (s, 3H), 3.47 (s, 3H), 3.78 $3.80(\mathrm{~m}, 4 \mathrm{H}), 4.10(\mathrm{t}, J=4 \mathrm{~Hz}, 2 \mathrm{H}), 4.18(\mathrm{t}, J=4 \mathrm{~Hz}, 2 \mathrm{H})$, 6.14 (s, 1H), 6.83 (s, 1H), 7.02 (s, 1H), 7.38 (d, $J=8 \mathrm{~Hz}$, 
2H), $7.88(\mathrm{~d}, J=8 \mathrm{~Hz}, 2 \mathrm{H}) ;{ }^{13} \mathrm{C} \mathrm{NMR}\left(\mathrm{CDCl}_{3}, 100 \mathrm{MHz}\right)$ $\delta: 21.59,59.06,59.10,68.73,69.34,70.32,70.67,101.05$, $101.26,106.48,107.29$, 128.25, 130.18, 131.66, 145.91, 146.60, 149.75, 153.97, 157.89, 161.27; IR (KBr) v: 2913, 1728, 1609,1564, 1507, 1384, 1164, 1045, 951, 853, 755, $555 \mathrm{~cm}^{-1}$; MS (ESI) $m / z: 465.1[\mathrm{M}+\mathrm{H}]^{+}$;

\subsection{9 目标化合物 $6 \mathrm{a} 5$ 和 $6 \mathrm{b5}$ 的合成}

$2.5 \mathrm{mmol}$ 化合物 7, $3.0 \mathrm{mmol}$ 间乙炔苯胺, $5.0 \mathrm{mmol}$ 无水碳酸钾和 $10.0 \mathrm{~mL}$ 无水乙醇, 升温至回流, TLC $[V($ 石油醚 $): V($ 乙酸乙酯 $)=1: 1]$ 检测至原料转化完毕 后停止反应, 减压蒸馏除去溶剂, 得到的固体水洗后过 滤, 即得纯产品 ${ }^{[27]}$.

4-(3-乙炔基苯胺基)-6,7-二甲氧基香豆素(6a5): 收 率 10.0\%. m.p. 273.0 275.0 ${ }^{\circ} \mathrm{C} ;{ }^{1} \mathrm{H}$ NMR (DMSO- $d_{6}$, $400 \mathrm{MHz}) \delta: 3.86(\mathrm{~s}, 6 \mathrm{H}), 4.28(\mathrm{~s}, 1 \mathrm{H}), 5.21(\mathrm{~s}, 1 \mathrm{H}), 7.00$ (s, 1H), $7.37 \sim 7.44(\mathrm{~m}, 4 \mathrm{H}), 7.66(\mathrm{~s}, 1 \mathrm{H}), 9.20$ (brs, 1H); ${ }^{13} \mathrm{C}$ NMR (100 MHz, DMSO- $\left.d_{6}\right) \delta: 61.09,61.29,86.56$, $87.75,88.05,105.43,109.11,111.11,127.89,130.53$, $132.69,133.91,135.01,143.84,150.52,154.19,157.48$, 157.62, 166.97; IR (KBr) v: 3284, 1650, 1535, 1503, 1437 , 1225, $800 \mathrm{~cm}^{-1}$; MS (ESI) m/z: $322.1[\mathrm{M}+\mathrm{H}]^{+}$; HRMS (ESI) calcd for $\mathrm{C}_{22} \mathrm{H}_{26} \mathrm{NO}_{6}[\mathrm{M}+\mathrm{H}]^{+}: 322.1001$, found 322.1081

4-(3-乙炔基苯胺基)-6,7-二(甲氧基乙氧)香豆素 (6b5): 收率 8.6\%. m.p. $193.0 \sim 195.0{ }^{\circ} \mathrm{C} ;{ }^{1} \mathrm{H}$ NMR $\left(\mathrm{DMSO}-d_{6}, 400 \mathrm{MHz}\right) \delta: 3.34(\mathrm{~s}, 6 \mathrm{H}) 3.70 \sim 3.73(\mathrm{~m}, 4 \mathrm{H})$, $4.17 \sim 4.20(\mathrm{~m}, 2 \mathrm{H}), 4.21 \sim 4.23(\mathrm{~m}, 2 \mathrm{H}), 4.29(\mathrm{~s}, 1 \mathrm{H})$, $5.23(\mathrm{~s}, 1 \mathrm{H}), 7.04(\mathrm{~s}, 1 \mathrm{H}), 7.37 \sim 7.52(\mathrm{~m}, 4 \mathrm{H}), 7.71(\mathrm{~s}$, $1 \mathrm{H}), 9.17$ (br s, $1 \mathrm{H}) ;{ }^{13} \mathrm{C}$ NMR (DMSO- $\left.d_{6}, 100 \mathrm{MHz}\right) \delta$ : 58.94, 68.93, 69.31, 70.72, 70.88, 82.18, 83.35, 83.78, $102.20,106.88,123.53,126.11,128.27,129.55,130.64$, $139.35,145.31,149.91,152.82,152.96,162.53$; IR (KBr) $v$ : $3256,1732,1646,1531,1376,1270,1119 \mathrm{~cm}^{-1}$; MS (ESI) $m / z: 410.1[\mathrm{M}+\mathrm{H}]^{+}$.

辅助材料(Supporting Information) 所合成化合物的 氢谱、碳谱和 GC-MS 等谱图数据. 这些材料可以免费 从本刊网站(http://sioc-journal.cn/)上下载.

\section{References}

[1] Wood-Kaczmar, A.; Gandhi, S.; Wood, N. W. Trends Mol. Med. 2006, 12, 521.
[2] Karhunen, T.; Tilgmann, C.; Ulmanen, I.; Julkunen, I.; Panula, P Histochem. J. Cytochem. 1994, 42, 1079.

[3] Hegazi, M. F.; Borchard, R. T.; Schowen, R. L. J. Am. Chem. Soc 1976, 98, 3048.

[4] Diamond, S. G.; Markham, C. H.; Treciokas, L. Ann. Neurol. 1978, 3, 267.

[5] Bartholini, G.; Pletscher, A. Pharmacol. Ther. 1975, 1, 407.

[6] Bonifácio, M. J.; Sutcliffe, J. S.; Torrão, L.; Wright, L. C.; Soares-da-Silva, P. Neuropharmacology 2014, 77, 334.

[7] Learmonth, D. A.; Bonifacio, M. J.; Soares-da-Silva, P. J. Med. Chem. 2005, 48, 8070 .

[8] Schrag, A. L. Neurology 2005, 4, 366.

[9] Learmonth, D. A.; Palma, P. N.; Vieira-Coelho, M. A.; Soares-da-Silva, P. J. Med. Chem. 2004, 47, 6207.

[10] Chen, D.; Wang, C. Y.; Lambert, J. D.; Ai, N.; Welsh, W. J.; Yang, C. S. Biochem. Pharmacol. 2005, 69, 1523.

[11] Backstrom, R.; Honkanen, E.; Pippuri, A.; Kairisalo, P.; Pystynen, J.; Heinola, K.; Nissinen, E.; Linden, I. B.; Mannisto, P. T.; Kaakkola, S.; Pohto, P. J. Med. Chem. 1989, 32, 841.

[12] Vladimir, G. Z.; Oenrich, I. P.; Pyodor, A. T. Tetrahedron 1994, $21,6377$.

[13] Zhang, T.; Zheng, Y.; Li, Y. F.; Wang, N. Chin. Chem. Lett. 2013, 24,719 .

[14] Backstrom, R.; Honkanen, E.; Pippuri, A.; Kairisalo, P.; Pystynen, J.; Heinola, K.; Nissinen, E.; Linden, I. B.; Mannisto, P. T.; Kaakkola, S.; Pohto, P. J. Med. Chem., 1989, 32, 841.

[15] Borgulya, J.; Bruderer, H.; Bernauer, K.; Zrcher, G.; Da-Prada, M. Helv. Chim. Acta 1989, 72, 952.

[16] Waldmeier, P. C.; Herdt, P. D.; Maitre. L. J. Neural Transm. Suppl. 1990, 32, 381.

[17] Ai, C. Z.; Wang, Y. H.; Li, Y.; Li, Y. H.; Yang, L. QSAR Comb. Sci. 2008, 27, 1183.

[18] Almeida, L.; Rocha, J. F.; Falcão, A.; Palma, P. N.; Loureiro, A. I.; Pinto, R.; Bonifácio, M. J.; Wright, L. C.; Nunes, T.; Soares-daSilva, P. Clin Pharmacokinet. 2013, 52, 139.

[19] Theodoros, S. S.; Konstantinos, E. L. Tetrahedron Lett. 2013, 54, 6517.

[20] Dasa, D. K.; Sarkara, S.; Khanc, K.; Belala, M.; Khan, A. T. Tetrahedron Lett. 2014, 55, 4869

[21] Tsuji, E.; Okazaki, K.; Takeda, K. Biochem. Biophys. Res. Commun. 2009, 378, 494

[22] Daly, J. W.; Benigni, J.; Minnis, R.; Kanaoka, R.; Witkop, B. Biochemistry 1965, 4, 2513.

[23] Sheng, R.; Lin, X.; Zhang, J.; Chol, K. S.; Huang, W. H.; Yang, B.; He, Q. J.; Hu, Y. Z. Bioorg. Med. Chem. 2009, 18, 6692.

[24] Jones, G. H.; Mackenzie, J. B. D.; Bertsonan, A.; Whalley, W. B. J. Chem. Soc. 1949, 562

[25] Xiao, D.; Martini, L. A.; Snoeberger, R. C.; III; Crabtree, R. C.; Batista, V. S. J. Am. Chem. Soc. 2011, 133, 9014.

[26] Braccio, M. D.; Grossi, G.; Roma, G.; Marzano, C.; Baccichetti, F.; Simonato, M.; Bordin, F. Farmaco 2003, 58, 1083.

[27] Kuroda, J.; Inamoto, K.; Hiroya, K.; Doi, T. Eur. J. Org Chem. 2009, 19, 2251.

[28] Zhang, L.; Meng, T. H.; Fan, R. H.; Wu, J. J. Org. Chem. 2007, 72, 7279 . 